\title{
QFEL: A NUMERICAL CODE FOR MULTI-DIMENSIONAL SIMULATION OF FREE ELECTRON LASERS IN THE QUANTUM REGIME
}

\author{
A. SCHIAVI ${ }^{1,2}$, N. PIOVELLA ${ }^{3,4}$, G.R.M. ROBB ${ }^{5}$ AND R. BONIFACIO ${ }^{3}$ \\ ${ }^{1}$ Dipartimento di Energetica, Università di Roma "La Sapienza" , \\ Via A.Scarpa 14, I-00161 Roma, Italy \\ E-mail: angelo.schiavi@uniroma1.it \\ 2 INFN-LNF, via E. Fermi 40, I-00044 Frascati, Roma, Italy \\ ${ }^{3}$ INFN-Sezione di Milano, via Celoria 16, I-20133 Milano, Italy \\ ${ }^{4}$ Dipartimento di Fisica, Università Degli Studi di Milano, \\ via Celoria 16, I-20133 Milano, Italy \\ 5 SUPA, Department of Physics, University of Strathclyde, \\ Glasgow, G4 ONG, Scotland, UK
}

\begin{abstract}
A new simulation tool has been developed for the simulation of the FEL equations in both the classical and quantum regimes to be applied to the investigation of proposed FEL models and to the exploration of parameter space for new experiments.
\end{abstract}

\section{Introduction}

Presently several projects are active in the research and development of new bright sources in the soft X-ray range using the free electron laser (FEL) mechanism $[1,2,3]$. Experiments are currently run or proposed to run in the self-amplified-spontaneous-emission (SASE) mode. One of the key disadvantages of SASE FEL is the large number of uncorrelated spikes in the pulse of emitted radiation, which also affects the spectrum of the FEL source [4]. A new regime of operation, called quantum SASE, could provide a dramatic improvement to the quality of the radiation extracted from an FEL [5]. For the realization of quantum SASE, the present paradigm of $\mathrm{GeV}$ electron bunches combined with a magnetic wiggler is not feasible 
due to the extremely long gainlength for this operation mode. It has been then proposed to test quantum SASE with an electromagnetic wiggler [6]. Detailed simulations of the quantum FEL model are necessary in order to address the feasibility of future experiments in this new regime.

\section{The 1d model equations}

The QFEL code is based on the one-dimensional quantum FEL model presented in [5]. The dynamics of the electron bunch is described by a Schrödinger equation for a matter-wave field $\Psi$ interacting with a pendulum potential proportional to the dimensionless radiation field amplitude $A$. The evolution of the radiation field is determined by the Maxwell equation for the $A$ field (in the slowly-varying envelope approximation, SVEA) interacting with the electron transverse current:

$$
\begin{gathered}
i \frac{\partial \Psi}{\partial \bar{z}}=-\frac{1}{2 \bar{\rho}^{3 / 2}} \frac{\partial^{2} \Psi}{\partial \theta^{2}}-i\left(A e^{i \theta}-A^{*} e^{-i \theta}\right) \Psi \\
\frac{\partial A}{\partial \bar{z}}+\frac{\partial A}{\partial z_{1}}=\int_{0}^{2 \pi} \frac{d \theta}{2 \pi}|\Psi|^{2} e^{-i \theta}+i \bar{\delta} A
\end{gathered}
$$

Here $\Psi\left(z_{1}, \bar{\delta}, \theta, \bar{z}\right)$ is a function of $z_{1}$, the coordinate along the electron bunch moving at the resonant velocity, of $\bar{\delta}$, the detuning parameter, of $\theta$, the electron phase, and of $\bar{z}$, the dimensionless wiggler length. The radiation amplitude $A\left(z_{1}, \bar{z}\right)$ is a function of the relative electron coordinate $z_{1}$ and of the position along the wiggler $\bar{z}$. The electron position $z_{1}$ is expressed in units of cooperation length $L_{c}$, whereas the wiggler position $\bar{z}$ is in units of gainlength $L_{g}$. For the definition of all these lengths and parameters, refer to $[5]$.

The quantum FEL parameter $\bar{\rho}$ represents the maximum number of photons emitted by a single electron as the bunch propagates through the wiggler. The value of $\bar{\rho}$ discriminates between the quantum and the classical regime. In the classical regime $(\bar{\rho} \gg 1)$, every electron emits and absorbs many photons in passing through the wiggler. In the quantum regime instead $(\bar{\rho}<1)$, the electron has a small probability of emitting just one photon in the whole process. This eventually leads to a completely different behavior in the quantum regime, where the above equations describe a two-level system. For solving numerically the quantum FEL equations, we chose to expand the $\Psi$ field into longitudinal momentum eigenstates $c_{n}$ 


$$
\Psi\left(z_{1}, \bar{\delta}, \theta, \bar{z}\right)=\sum_{n} c_{n}\left(z_{1}, \bar{\delta}, \bar{z}\right) e^{i n \theta},
$$

so that the equations to solve become

$$
\begin{gathered}
\frac{\partial c_{n}}{\partial \bar{z}}=-i\left(\frac{n^{2}}{2 \bar{\rho}^{3 / 2}}+n \bar{\delta}\right) c_{n}-\left(A c_{n-1}+A^{*} c_{n+1}\right) \\
\frac{\partial A}{\partial \bar{z}}+\frac{\partial A}{\partial z_{1}}=b\left(z_{1}, \bar{z}\right)
\end{gathered}
$$

where the bunching factor $b$ is computed using a weight function $G(\bar{\delta})$, which is a normalized Gaussian distribution centered on the mean detuning parameter $\bar{\delta}_{0}$ :

$$
b\left(z_{1}, \bar{z}\right)=b_{n} \sum_{n} \int d \bar{\delta} G(\bar{\delta}) c_{n} c_{n-1}^{*}
$$

The normalization factor $b_{n}$ takes into account the dimensionality of the simulation, as described later on in this paper.

\section{Higher dimensions and transverse terms}

The principal aim of developing the QFEL code was to extend the previous analysis and simulation work carried out in one longitudinal dimension by adding the transverse dimensions and introducing a description of the transverse FEL dynamics. As a consequence, the electron field $\Psi\left(z_{1}, \bar{x}, \bar{y}, \bar{\delta}, \theta, \bar{z}\right)$ and the radiation amplitude $A\left(z_{1}, \bar{x}, \bar{y}, \bar{z}\right)$ acquired two transverse coordinates, namely $\bar{x}$ and $\bar{y}$, expressed in units of the initial width of the bunch in the transverse plane. The beam profile had a Gaussian profile along each transverse direction, with the possibility of simulating axis-symmetric beams $\left(\sigma_{x}=\sigma_{y}\right)$, and beams with an oblate transverse profile $\left(\sigma_{x} \neq \sigma_{y}\right)$. The effects due to beam emittance and to the profile of a laser wiggler will be discussed in a forthcoming work.

\section{Numerical scheme}

In order to integrate the quantum FEL equations, the phase space was sampled on a discrete grid and the dynamical variables, namely $A$ and the $c_{n}$ states, were evaluated on this grid. A combination of different finite difference (FD) schemes were adopted in an operator splitting algorithm. 


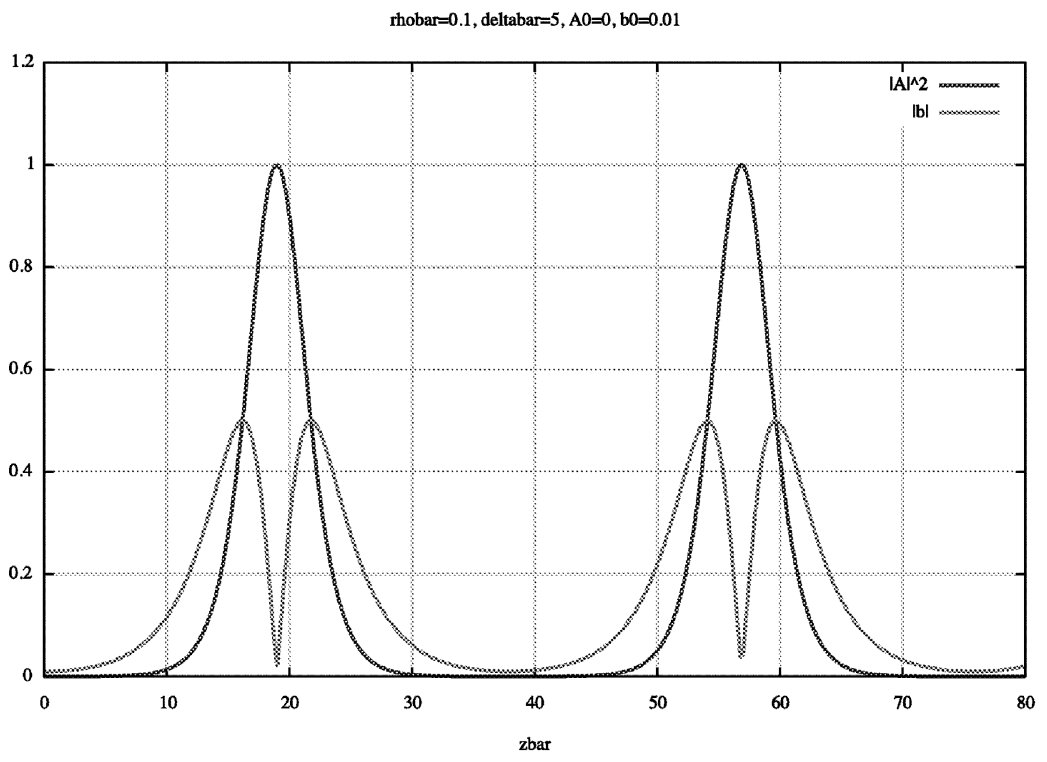

Figure 1. 1d steady-state simulation in the quantum regime $(\bar{\rho}=0.1)$ : normalized radiation intensity $|A|^{2}$ and bunching factor $b$ as a function of the position $\bar{z}$ along the wiggler.

An explicit FD scheme coupled to a Runge-Kutta stepper was used for integrating the interaction of the field and the electron bunch [8]. A cubicinterpolation propagation (CIP) method was implemented for solving the advection equation of the radiation field in the electron bunch rest frame [9]. The high dimensionality of the simulation phase space (up to $N=5$ ) and the large number of unknowns (typically from $10^{6}$ to $10^{8}$ ) demanded for a parallel implementation of the code. To this end we adopted a fixed linear domain decomposition technique using a master-slaves paradigm. The whole code has been written in F90 using the MPI library.

\section{Quantum 2d steady-state}

To illustrate some typical effects studied using QFEL, we present here the 1d and $2 \mathrm{~d}$ steady-state evolution in the quantum regime. These results were reproduced to test the high-gain FEL dynamics. It is important to note that for the simulation of SASE FEL the radiation propagation is an essential ingredient. The simulations were carried out for $\bar{\rho}=0.1$ and with a mean detuning centered on the quantum resonance, i.e. $\bar{\delta}_{0}=5$. The initial field amplitude was set to zero, $A_{0}=0$, whilst the beam was initialized in a state with bunching $b_{0}=0.01$. Such a small value served as a seed to initiate the 
beam-radiation interaction. The one-dimensional case, see figure 1 , shows the typical quantum FEL evolution for the steady-state regime, were the electrons swing back and forth from the initial momentum state $c_{0}$ to the first recoil state $c_{-1}$. Trace of this mechanism remains in the cyclic pulsing of the radiation intensity $|A|^{2}$. The peak value of the intensity is equal to 1 as implied by the quantum scaling used in the FEL equations.

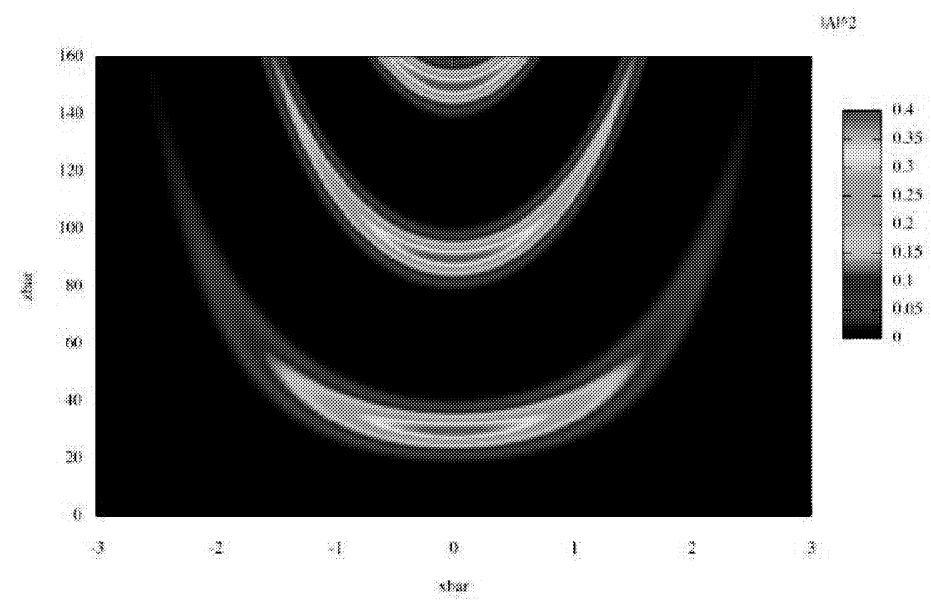

Figure 2. $2 \mathrm{~d}$ steady-state simulation in the quantum regime $(\bar{\rho}=0.1)$ : transverse radiation intensity $|A|^{2}$ streaked vertically along the wiggler position $\bar{z}$.

Figure 2 presents a $2 \mathrm{~d}$ simulation for the same initial parameters, with the exception that the beam has now a finite transverse width. The figure shows the field intensity map streaked vertically along the wiggler. No transverse terms are switched on here, so that each part of the beam evolves independently from the others. The peak intensity is reached on axis $(\bar{x}=$ 0 ) and the maximum value is reduced to 0.4 . The gain length and the lethargy period are longer compared to the $1 \mathrm{~d}$ case. This is because the bunching factor $b$, which is the source term for the radiation field, is a function of the local electron "density". Hence the wings of the beam effectively see a reduced bunching factor with respect to the center of the beam and evolve on a longer scalelength.

The effects of radiation diffraction were then included in the model by 
adding a term proportional to the transverse laplacian of the radiation field $A$ :

$$
\frac{\partial A}{\partial \bar{z}}+\frac{\partial A}{\partial z_{1}}-i a \nabla_{\perp}^{2} A=b\left(z_{1}, \bar{x}, \bar{z}\right)
$$

where $a$ is an adimensional parameter [7]. Figure 3 shows the effects of radiation diffraction: the intensity of the radiation reduces even more with respect to the $1 \mathrm{~d}$ case, because the field can now escape in the transverse direction and is not further amplified by the interaction with the electrons.

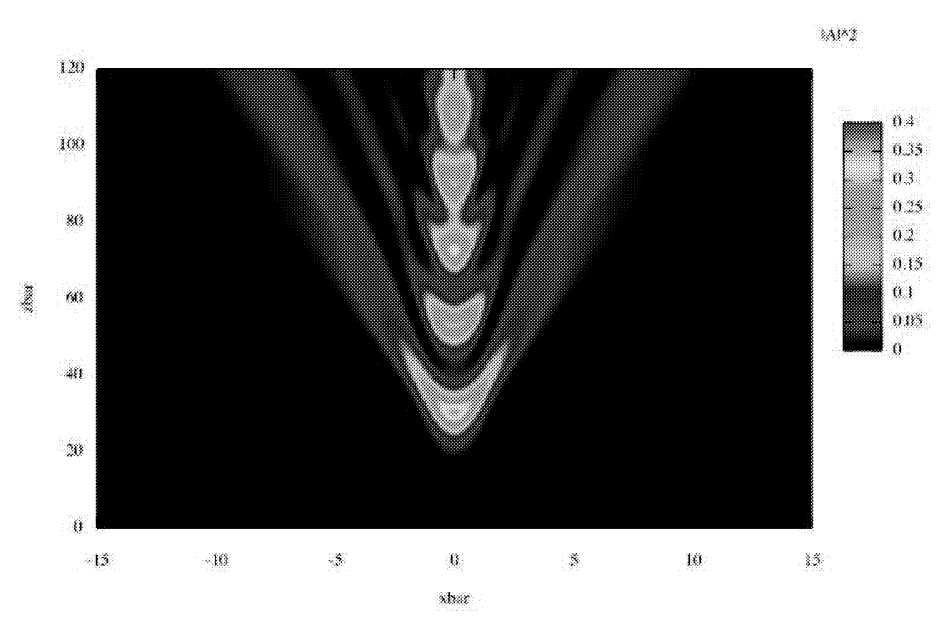

Figure 3. 2d steady-state simulation in the quantum regime $(\bar{\rho}=0.1)$ with radiation diffraction: transverse radiation intensity $|A|^{2}$ streaked vertically along the wiggler position $\bar{z}$.

\section{Normalization and Scaling}

The electron field is slice-normalized for each $z_{1}$ to the constant current profile $I_{0}\left(z_{1}\right)$

$$
\int d \bar{x} \int d \bar{y} \int d \bar{\delta} \int_{0}^{2 \pi} \frac{d \theta}{2 \pi}\left|\Psi\left(z_{1}, \bar{x}, \bar{y}, \bar{\delta}, \theta ; \bar{z}\right)\right|^{2}=I_{0}\left(z_{1}\right) .
$$

Since the Maxwell equation for $A$ is quadratic in the electron field, it not possible to renormalize the Schrödinger equation for the electron beam 
without affecting the source term in the Maxwell equation. The dynamics of $1 \mathrm{~d}, 2 \mathrm{~d}$ and $3 \mathrm{~d}$ models is intrinsically different due to the spreading out of the electron wavefunction, even in absence of transverse coupling terms. This leads to different gain and peak intensity values depending on the dimensionality of the simulation. In order to recover consistency between the three models, a renormalization factor $b_{n}$ has been introduced in equation 6 , which carries the information about the number of dimensions. The complete $3 \mathrm{~d}$ model was taken as a reference, whereas the $1 \mathrm{~d}$ e $2 \mathrm{~d}$ models were rescaled in order to match the gain of the $3 \mathrm{~d}$ equations. Effectively this corresponds to considering the $1 \mathrm{~d}$ model as a description of just the inner core of the beam, whilst the $2 \mathrm{~d}$ equations are those for a longitudinal slice of the beam. As a consequence the gain of the $1 \mathrm{~d}$ model and of the central part of the $2 \mathrm{~d}$ model are equal to the gain of the axial part of the beam in the fully $3 \mathrm{~d}$ model. Clearly the total radiation intensity, integrated over the transverse directions, becomes smaller going from $3 \mathrm{~d}$ to $1 \mathrm{~d}$, as just a part of the whole beam is considered. These observations are summarized in figure 4 , which presents the log plot of the integrated field intensity for the three models when the renormalization factor $b_{n}$ was introduced.

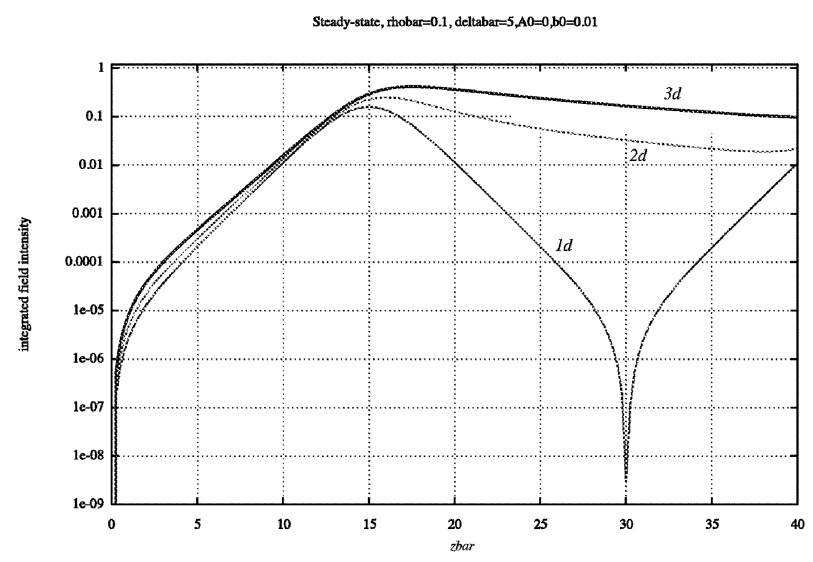

Figure 4. Comparison of the integrated radiation intensity as a function of position $\bar{z}$ along the wiggler for the $1 \mathrm{~d}$ (red), $2 \mathrm{~d}$ (green) and $3 \mathrm{~d}$ models (blue).

\section{SASE regime and grid-independent noise}

One of the most fundamental requirements in addressing the accuracy and stability of a numerical simulation is that the solution to the dynamical 
equations is not dependent on the choice of a particular discretization of the integration domain. It is therefore common practice to refine the simulation mesh until the numerical solution stabilizes. This condition often represents the best compromise, balancing the accuracy of the computation and the resources (memory and CPU time) required to achieve it. Several criteria exist for monitoring the stability of a particular numerical scheme (e.g. time-step limiters for implicit or explicit FD schemes), but it is only through a trial-and-error process that the stability of the final solution can be addressed. It is important to note that in a non-linear system, as the one solved by QFEL, this process as to be repeated for every choice of the dynamical parameters (in our case the most sensible one is clearly the $\bar{\rho}$ parameter). In the simulation of the SASE operation, we included the radiation propagation, and the electron bunch was initialized with a small bunching (usually $b<0.01$ ) and with a random phase $e^{i \phi}$. The phase $\phi\left(z_{1}, y, z\right)$ is a function of the point coordinate and is used to cancel correlations between the different parts of the electron beam. Instead of just sampling the random phase on the grid points, we developed an algorithm for generating a noise function in the Fourier-transformed space and remapping it onto the simulation grid. This allowed us to set a particular level of phase noise, controlling amplitude and waveform in a grid-independent way. It was then possible to check the stability of the simulation as described at the beginning of this section. Figure 5 presents the same pseudo-random phase mapped to a coarse mesh and to a finer one, where the grid has 5 times more points.
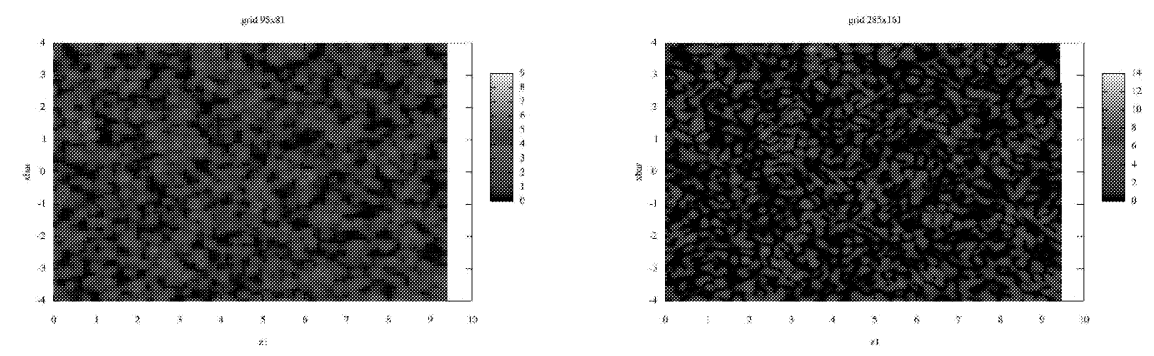

Figure 5. Random noise for a bidimensional simulation: coarse grid (left) and fine grid (right). 


\section{Conclusions}

A new tool, the QFEL code, has been developed for studying quantum FEL systems in a full $3 \mathrm{~d}$ spatial geometry. The choice of a $3 \mathrm{~d}$ cartesian model over a $2 \mathrm{~d}$ cylindrical geometry was motivated by the necessity of simulating the start-up from noise in the SASE operation mode. To this end, initializing a random annular phase, as in the $2 \mathrm{~d}$ cylindrical case, would have introduced unwanted correlations far from the actual beam conditions. As a consequence, the model equations required up to 100 million grid points for the accurate description of the FEL dynamics. To tackle such a large number of unknowns, the numerical scheme was coded to run on a parallel computer using standard and efficient software solutions. The QFEL code can be easily run in 1, 2 or 3 spatial dimensions in a consistent way. Therefore feasibility studies of proposed experiments can be carried out in lower dimensions, which require less time and computing resources. Fine tuning of the parameters and exploration of the details of the full dynamical content of the model equations can then be obtained by going to higher dimensions, including transverse terms and propagation effects.

\section{Acknowledgments}

G.R.M. Robb acknowledges support from the Leverhulme Trust (grant F00273I).

\section{References}

1. R. Bonifacio, C. Pellegrini, L.Narducci, Opt. Commun. 50, 373 (1984).

2. J. Andruszkow et al.,Phys. Rev. Lett. 85, 385 (2000).

3. Linac Coherent Light Source (LCLS) Conceptural Design Report, SLAC Report No. SLAC-R-593, (2002).

4. R. Bonifacio, L. De Salvo, P. Pierini, N. Piovella and C. Pellegrini, Phys. Rev. Lett. 73, 70 (1994).

5. R. Bonifacio, N. Piovella and G.R.M. Robb, Nucl. Inst. and Meth A 543, 645 (2005).

6. R. Bonifacio, Nucl. Inst. and Meth A 546, 634 (2005).

7. E.T. Scharlemann et al.,Phys. Rev. Lett. 54, 1925 (1985).

8. W.H. Press and S.A. Teukolsky and others, Numerical Recipes in C - The Art of Scientific Computing - Second Edition, Cambridge University Press, USA (1994).

9. T. Yabe and T. Aoki, Comp. Phys. Commun. 66, 219 (1991). 